\title{
High-speed identification of suspended carbon nanotubes using Raman spectroscopy and deep learning
}

\author{
Jian Zhang ${ }^{1}$, Mickael L. Perrin (10 ${ }^{1 凶}$, Luis Barba², Jan Overbeck,3, Seoho Jung ${ }^{4}$, Brock Grassy $\mathbb{C}^{2}$, Aryan Agal ${ }^{2}$, Rico Muff', \\ Rolf Brönnimann ${ }^{1}$, Miroslav Haluska ${ }^{4}$, Cosmin Roman ${ }^{4}$, Christofer Hierold ${ }^{4}$, Martin Jaggi ${ }^{2}$ and Michel Calame $\mathbb{B}^{1,3 凶}$
}

\begin{abstract}
The identification of nanomaterials with the properties required for energy-efficient electronic systems is usually a tedious human task. A workflow to rapidly localize and characterize nanomaterials at the various stages of their integration into large-scale fabrication processes is essential for quality control and, ultimately, their industrial adoption. In this work, we develop a high-throughput approach to rapidly identify suspended carbon nanotubes (CNTs) by using high-speed Raman imaging and deep learning analysis. Even for Raman spectra with extremely low signal-to-noise ratios (SNRs) of 0.9, we achieve a classification accuracy that exceeds $90 \%$, while it reaches $98 \%$ for an SNR of 2.2. By applying a threshold on the output of the softmax layer of an optimized convolutional neural network $(\mathrm{CNN})$, we further increase the accuracy of the classification. Moreover, we propose an optimized Raman scanning strategy to minimize the acquisition time while simultaneously identifying the position, amount, and metallicity of CNTs on each sample. Our approach can readily be extended to other types of nanomaterials and has the potential to be integrated into a production line to monitor the quality and properties of nanomaterials during fabrication.
\end{abstract}

\section{Introduction}

Because of their nanoscale diameter and high carrier mobility, carbon nanotubes (CNTs) are prominent among a variety of nanoscale materials that have been considered for next-generation energy-efficient electronic systems ${ }^{1}$. For example, CNTs have been successfully used for chemical and physical sensing at unprecedented low power consumption in the range of $\mu \mathrm{W}$ per sensor function ${ }^{2,3}$. Recently, a beyond-silicon modern microprocessor was designed and fabricated entirely using CNT field-effect transistors (CNFETs $)^{4}$. On the road to the

\footnotetext{
Correspondence: Mickael L. Perrin (mickael.perrin@empa.ch) or Michel Calame (michel.calame@empa.ch)

'Laboratory for Transport at Nanoscale Interfaces, Empa, Swiss Federal Laboratories for Materials Science and Technology, CH-8600 Dübendorf, Switzerland

${ }^{2}$ Machine Learning and Optimization Laboratory, School of Computer and Communication Sciences, EPFL, CH-1015 Lausanne, Switzerland Full list of author information is available at the end of the article These authors contributed equally: Jian Zhang, Mickael L. Perrin.
}

commercialization of CNT-based electronics ${ }^{1,5,6}$, methods to rapidly characterize CNTs at various stages of largescale fabrication processes are essential for quality control and for the industrial adoption of CNFET technology. In particular, Raman spectroscopy has the advantages of being a nondestructive, noncontact approach, providing chemical, and structural information with micrometer spatial resolution ${ }^{7}$. In addition, the position, width, and shape of the characteristic peaks in the Raman spectra are indicators of the molecular or crystalline structure, electronic properties, and the quality of the materials. In the case of CNTs, the so-called G band shape and resonant electronic Raman scattering are used to distinguish the type of $\mathrm{CNT}^{8-10}$. (see Supplementary information Fig. S1)

Due to the low efficiency of Raman scattering, subtle spectral signals are easily masked by background noise ${ }^{8,11}$. As a result, Raman imaging at high speed has been technically challenging because Raman spectroscopy usually requires relatively high power (in the milliwatt

\section{(c) The Author(s) 2022}

(c) (i) Open Access This article is licensed under a Creative Commons Attribution 4.0 International License, which permits use, sharing, adaptation, distribution and reproduction cc) in any medium or format, as long as you give appropriate credit to the original author(s) and the source, provide a link to the Creative Commons license, and indicate if changes were made. The images or other third party material in this article are included in the article's Creative Commons license, unless indicated otherwise in a credit line to the material. If material is not included in the article's Creative Commons license and your intended use is not permitted by statutory regulation or exceeds the permitted use, you will need to obtain permission directly from the copyright holder. To view a copy of this license, visit http://creativecommons.org/licenses/by/4.0/. 
a

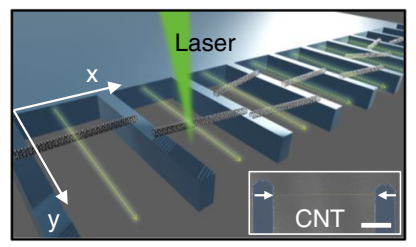

High-speed raman imaging

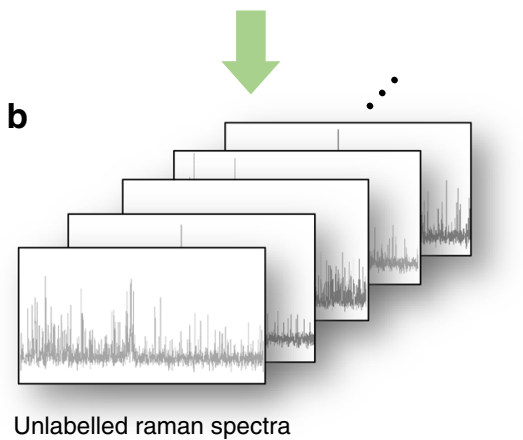

C

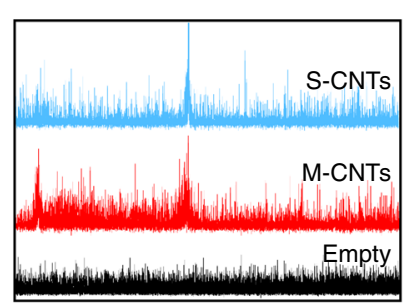

Large labeled datasets

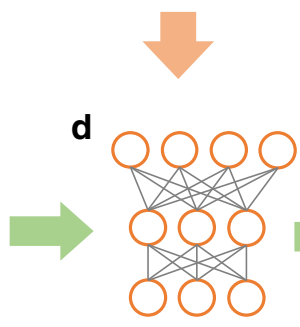

Deep learning model f

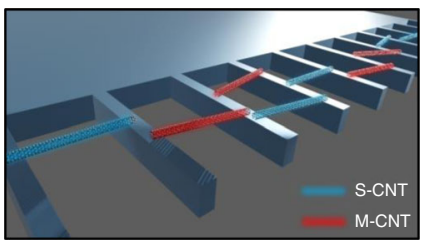

SWNT prediction

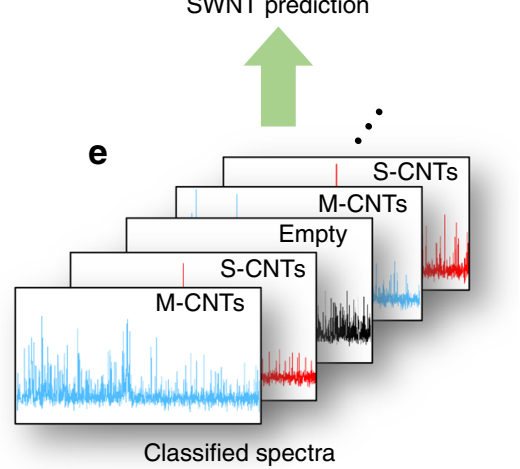

Fig. 1 Schematic illustration of deep learning-based Raman spectra analysis for CNT identification. a Implementation of high-speed Raman imaging on a fork-like growth substrate. b Generation of unlabeled Raman spectra. c Large labeled datasets organized into three classes: S-CNTs, MCNTs, and empty. d Deep learning model. e Classification of individual spectra using the model. $\mathbf{f}$ CNT identification.

range) and long exposure times (hundreds of milliseconds to a few seconds) to reach the desired identification accuracies. This issue can be partially overcome by collecting Raman-scattered photons using a fast electronmultiplying charge-coupled device (EMCCD), improving the signal-to-noise ratio (SNR) at scanning speeds below 1 millisecond per spectrum ${ }^{12,13}$. Nevertheless, at such low integration times, achieving a reliable distinction between different types of CNTs is challenging.

Machine-learning (ML) algorithms may offer a solution, as they have recently been used to solve complex recognition and classification problems, such as image ${ }^{14}$ and facial recognition ${ }^{15,16}$, as well as speech ${ }^{17,18}$ and text understanding $^{19,20}$. Their underlying strength is their excellence at recognizing patterns, either by relying on previous experience (supervised ML) or without any a priori knowledge of the system itself (unsupervised $M L)^{21,22}$. The number of tasks addressed by $M L$ is growing every day, with many applications in image processing, e.g., digital pathology ${ }^{23}$, self-driving $\operatorname{cars}^{24}$, or image enhancement ${ }^{15}$. Of particular interest for classification purposes are artificial neural networks, networks of interconnected neurons that are trained on datasets of known classes to find patterns that distinguish these classes as well as possible. In recent years, deep neural networks consisting of multiple layers of various types (fully connected, convolutional, max pooling, dropout, etc.), have become the dominant tool in ML due to the remarkable results they have achieved ${ }^{14-20}$. Convolutional neural networks (CNNs), consisting mostly of convolutional layers, have been widely applied in computer vision for image classification or object recognition and are also playing an increasingly important role in the analysis of spectroscopic data, including Raman spectra ${ }^{11,25}$.

Here, we develop a high-throughput approach to rapidly identify suspended CNTs based on the combination of deep learning, high-speed Raman spectroscopy, and an optimized scan strategy. We apply our approach to suspended CNTs that were grown on fork-like growth substrates that are optimized for a high-throughput dry transfer process. Such a process has been demonstrated to result in clean and CMOS-compatible CNT integra$\operatorname{tion}^{26,27}$ and has been used for ultralow-power gas sensors $^{2,3}$ nanoresonators ${ }^{28}$, and quantum device applications ${ }^{29,30}$. The workflow of the identification is schematically depicted in Fig. 1. Individual CNTs are grown and suspended across $\mathrm{SiO}_{2} / \mathrm{Si}$ forks (see Fig. 1a). To achieve a high-throughput approach, we implement a line scan at each fork instead of using the time-consuming full-mapping method. We optimize the line-scan parameters to obtain sufficient spectral information while allowing for unambiguous localization of CNTs and classification into 'M-CNTs' 'S-CNTs', or 'Empty' (see methods). All the (unlabeled) Raman spectra obtained from the line scan (see Fig. 1b) are then classified individually using an ML model (see Fig. 1d), which is trained on a large collection of labeled Raman spectra (Fig. 1c). After the training was completed, new individual Raman 
a

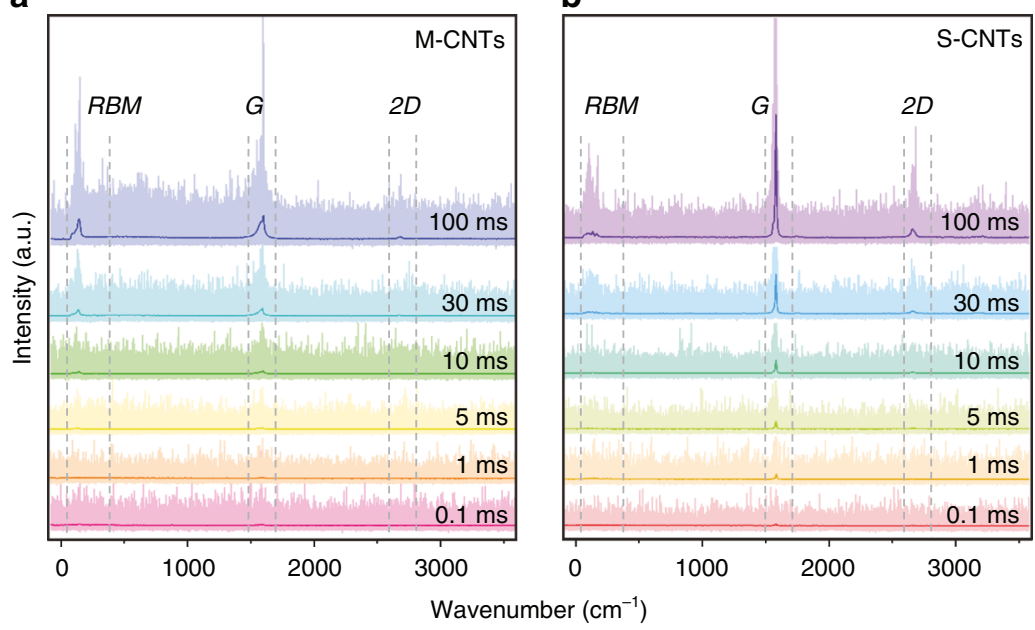

C

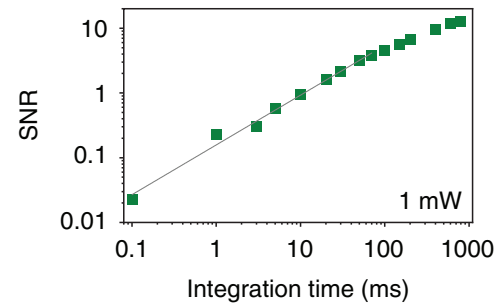

d

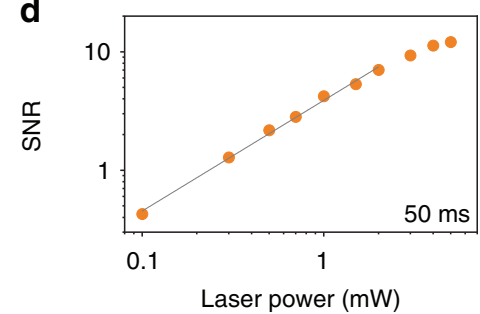

Fig. 2 Raman analysis of CNTs. a Raman spectra of M-CNTs with various integration times at fixed power ( $1 \mathrm{~mW}$ ). At each setting, the average of 1250 spectra from 32 M-CNTs are shown in bold and overlaid on these single spectra. Spectra are colored according to integration time. $\mathbf{b}$ Raman spectra from $30 \mathrm{~S}$-CNTs, with the same data size and plotting formats as in a. c SNR versus integration time at fixed power (1 mW). d SNR versus laser power at a fixed integration time $(50 \mathrm{~ms})$.

spectra were classified using the trained machine-learning model (Fig. 1e). To improve the classification accuracy, we apply additional methods. First, we apply a threshold on the output of the softmax layer to reduce the number of false positives and the number of false negatives. Second, we developed an optimized scanning strategy that rescans areas of interest and identifies the position and amount of CNTs across each fork (Fig. 1f) with increased efficiency compared to line scans. We elaborate on each of these steps in the remainder of this article.

\section{Results and discussion Dataset generation}

To minimize the scan time of each fork, the Raman spectra need to be acquired using an integration time as short as possible. Short integration times lead to low SNR and a reduction in the classification accuracy of the machine-learning model. Shorter integration times can be compensated by higher laser intensities up to the point where damage to the CNTs occurs. To investigate this trade-off between speed and accuracy and to identify the optimum settings, we built a range of datasets consisting of Raman spectra acquired using different integration times (ranging from 1 to $600 \mathrm{~ms}$ at $1 \mathrm{~mW}$ power) and different laser powers (ranging from 0.1 to $3 \mathrm{~mW}$ for an integration time of $50 \mathrm{~ms}$ ) on suspended CNT samples. An ML model was trained on the training dataset and tested on a separately acquired validation dataset, acquired in a similar fashion as the training dataset. To train the ML model, we constructed a large-scale labeled dataset consisting of 62,130 spectra at 19 Raman settings with different integration times and laser powers, acquired on 21 trenches containing CNTs with Raman characteristics of metallic CNTs (M-CNTs), 20 trenches containing CNTs with Raman characteristics that do not correspond to typical metallic CNTs (S-CNTs), and 20 trenches where no CNTs were found. In addition, we separately constructed labeled datasets for the validation step consisting of 48,887 spectra acquired on 10 trenches containing S-CNTs, 11 trenches containing M-CNTs, and 15 trenches where no CNTs were present. Figure $2 \mathrm{a}$ and $\mathrm{b}$ shows the average spectra acquired at various integration times for S-CNTs and M-CNTs. The spectra acquired at various laser powers for S-CNTs and M-CNTs are shown in Supplementary Information Fig. S2. The plots also display the overall envelope of all spectra (1250 spectra for each setting) as a shaded background. Here, the overall spread is significantly larger than for an averaged spectrum, highlighting the large spectrum-to-spectrum variability. Additionally, for the lower integration times, the SNR is drastically reduced. We quantified the SNR, and we show its dependence on the integration times and laser power in Fig. 2c and d. As expected, in both plots, the SNR scales linearly with integration times and laser power. We note that an SNR below 1 implies that the signal cannot be distinguished from noise, as is the case for all integration times below $10 \mathrm{~ms}$ at $1 \mathrm{~mW}$ and $0.1 \mathrm{~mW}$ with $50 \mathrm{~ms}$.

\section{Training and validation of the neural network}

The neural network architecture used for classification (see Fig. 3a) was obtained using a gridsearch through 

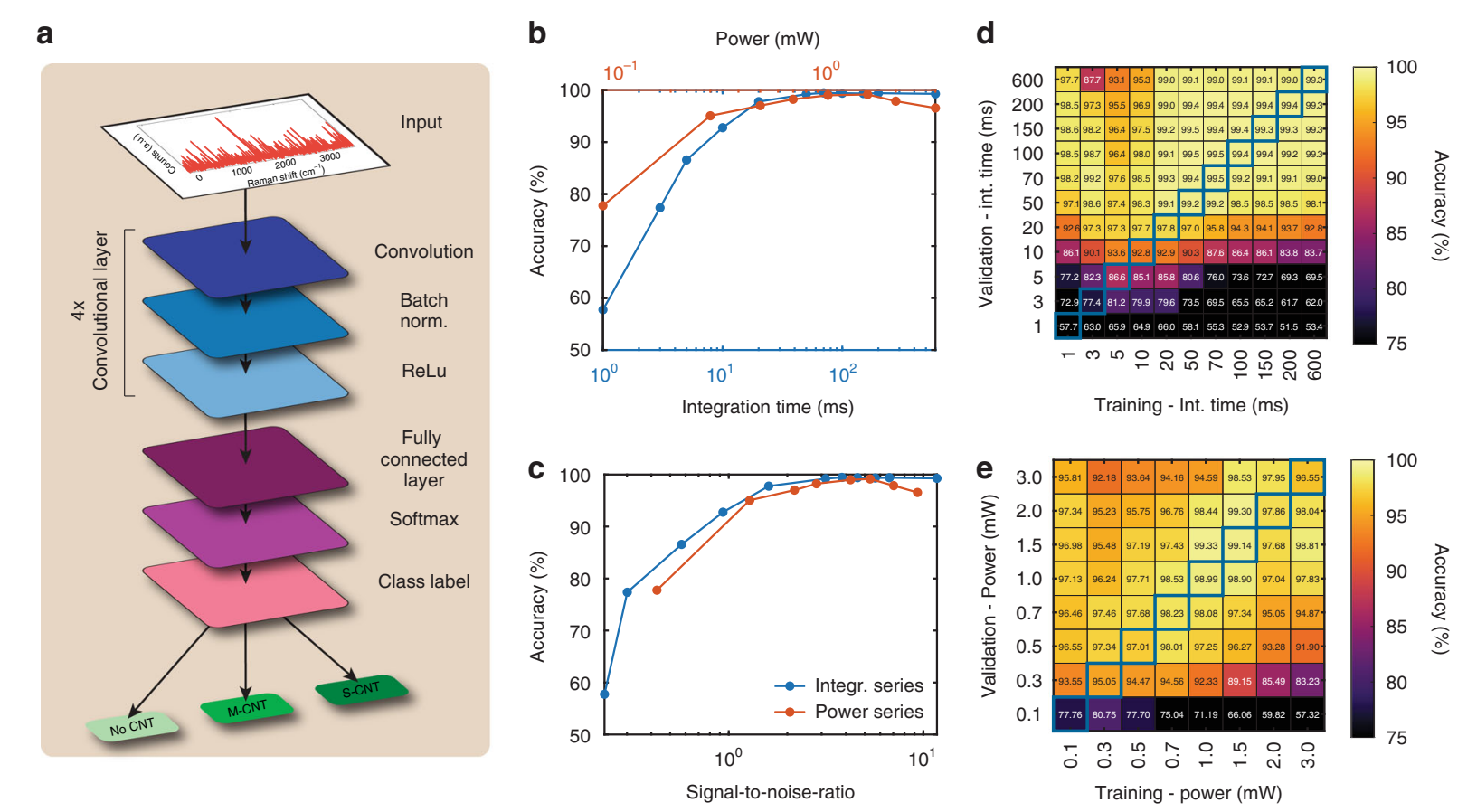

Fig. 3 Training and validation of the deep learning model. a Architecture of the convolutional neural network. $\mathbf{b}, \mathbf{c}$ Accuracy of the model while varying the integration times used for training and validation. $\mathbf{d}$, e Accuracy of the model while varying the laser power used for training and validation.

various neural network architectures (see Supplementary information: Part 2). The optimal network was found to be a convolutional neural network (CNN) (Fig. 3a), with 4 convolutional layers followed by fully connected layers, a softmax layer, and finally a cross-entropy layer for defining the loss function. More details about the network architecture are provided in the Methods section. To explore the characteristic bands extracted by the model, we plotted the activation values for the first convolutional layer in the plots below for a randomly selected spectrum acquired on semiconducting and metallic CNTs, as shown in Supplementary Information Fig. S3. The results are in agreement with the Raman-active CNT peaks and corroborate the fact that the network is activated by the characteristic spectral features.

The classification accuracies obtained for datasets with different integration times and laser powers are presented in Fig. 3b. The accuracy is $\sim 60 \%$ for the shortest integration time $(0.1 \mathrm{~ms})$, increases to $>90 \%$ at $10 \mathrm{~ms}$ and reaches $>98 \%$ for $>30 \mathrm{~ms}$. The accuracy for the power dependence starts at $78 \%$ for $0.1 \mathrm{~mW}$ and reaches a maximum of $99 \%$ at $1 \mathrm{~mW}$, after which it slightly decreases to $96 \%$. This decrease is likely caused by heating or damage from the intense laser irradiation of the $\mathrm{CNTs}^{31,32}$. Figure $3 \mathrm{c}$ presents the same accuracies, plotted against the SNRs obtained in Fig. 2c and d.

Furthermore, we explore the generalization of a model trained on specific Raman settings (power and integration time) and tested on data acquired with different settings. This analysis is relevant for investigating the accuracy of the model in conditions in which the test data do not match the same acquisition process as the training data. This could, for instance, be due to the drift of the experimental parameters or other temporary limitations arising during the characterization process. For completeness, for each integration time at which the model is trained, the accuracy for all integration times is obtained, yielding a matrix of all accuracies (see Fig. 3d), with the parameters used for training on the horizontal axis and those for validation on the vertical axis. The same procedure is repeated for power dependence (see Fig. 3e). We note that the highlighted accuracies on the diagonal of the matrix correspond to identical training and validation parameters and are identical to those presented in Fig. 3b. We find that the model is remarkably robust against variation. Additionally, models trained on low SNR data perform well on higher SNR data, but not the opposite. In fact, models trained on high SNR datasets perform poorly on low SNR datasets. For varying integration times, accuracies of $>90 \%$ are reached when testing on $20 \mathrm{~ms}$ data, independent of the testing parameters, while for testing and training times $>50 \mathrm{~ms}$, all combinations yield $>98 \%$. We note several outliers in the plot (such as training $3 \mathrm{~ms}$ and validation $600 \mathrm{~ms}$, which we attribute to outliers in the dataset). Nevertheless, the overall trend is robust. For the power dependence, we find that the 
a

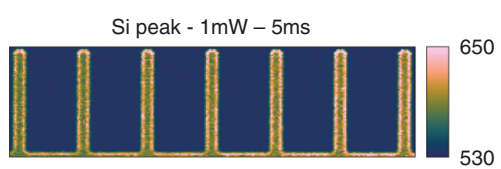

b

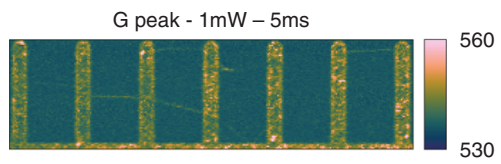

C
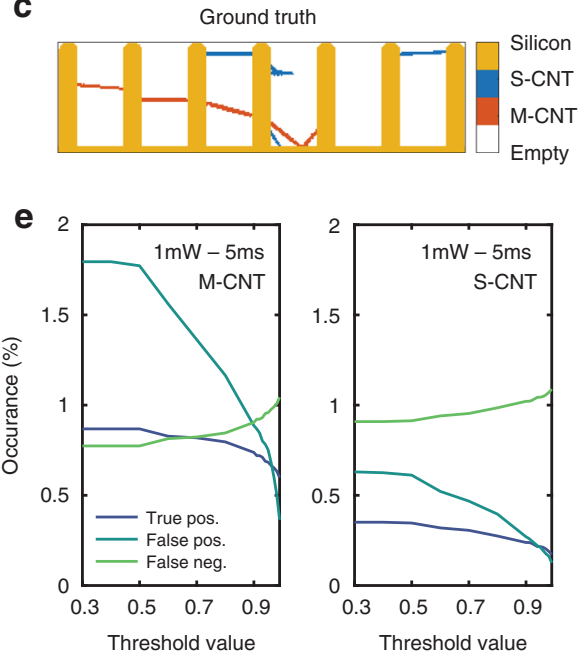
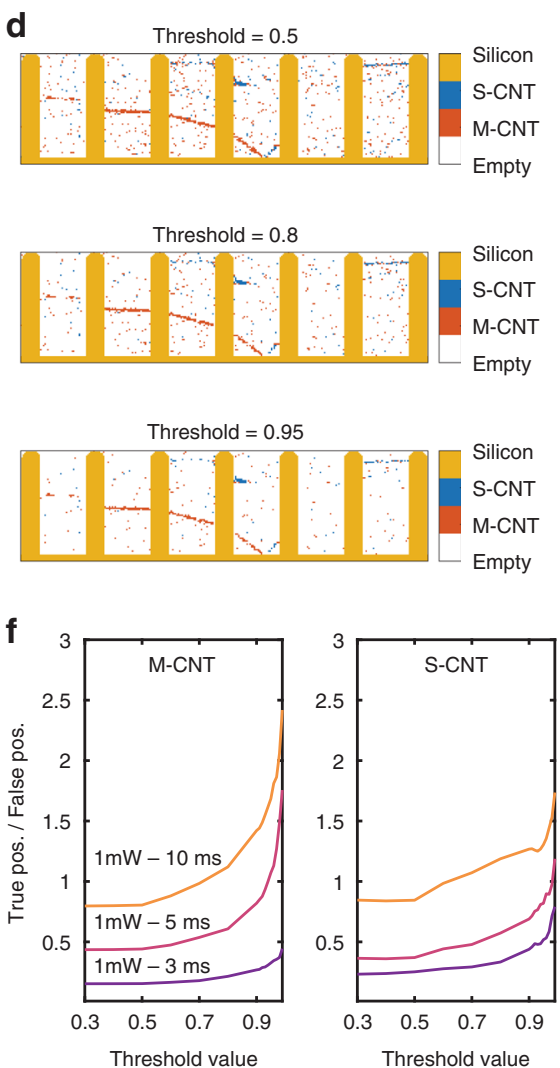

Fig. 4 Classification of a Raman map for varying threshold values. a Raman intensity map of the Silicon peak. $\mathbf{b}$ Raman intensity map of the G-peak. c Ground truth map. d Map of the predicted classes for varying softmax threshold values. e Occurrence of true positives, false positives, and false negatives for varying softmax threshold values for M-CNTs and S-CNTs. $\mathbf{f}$ Ratio of false-positive/true positives for varying softmax threshold values for M-CNTs and S-CNTs.

accuracy is the highest close to the diagonal and decreases when moving away from it. Again, we find that models trained on low SNR datasets perform well on high SNR datasets, but not vice versa.

\section{Softmax thresholding}

With the deep learning model being trained and its accuracy assessed, we proceed with its application to a map acquired on a series of forks. Figure $4 \mathrm{a}$ and $\mathrm{b}$ shows the intensities of the silicon $\left(520 \mathrm{~cm}^{-1}\right)$ and G-peak $\left(\sim 1580 \mathrm{~cm}^{-1}\right)$, respectively, obtained from a Raman map acquired at a low integration time of $5 \mathrm{~ms}$ and a laser power of $1 \mathrm{~mW}$ focused on CNTs with an objective magnification of $\times 50$ and NA of 0.55 . Figure $4 c$ presents the ground truth established using Raman spectra acquired with integration times of $800 \mathrm{~ms}$ and $1 \mathrm{~mW}$. We note that the silicon labels do not originate from the deep learning model but are obtained by applying a thresholding function to the intensity of the silicon peak and that there may be CNTs that are undetectable with the laser wavelength used. To increase the confidence of the classification by the neural network for fast map scans, we apply a threshold to the output of the softmax layer. This means that the S-CNT and M-CNT classes are only used if the confidence of the network for these classes exceeds a predefined threshold value. If this is not the case, the pixel in question is classified as Empty. Figure $4 d$ shows the fast map with each pixel classified for three selected threshold values. The plot for a threshold of 0.5 qualitatively reproduces the ground truth, albeit with a large amount of noise (randomly distributed pixels that are misclassified). For an increasing threshold, the noise gradually decreases, as does-to a lesser extent-the number of correctly classified pixels. To quantify the efficacy of the thresholding approach, Fig. 4e shows the number of true positives (TP), false positives (FP), and false negatives (FN) for M-CNTs and S-CNTs as a function of the threshold value. The threshold ensures that only the S/M pixels with a high classification fidelity (value at softmax layer) remain in the $\mathrm{S} / \mathrm{M}$ class. Increasing this threshold therefore drastically reduces the number of FPs, which usually have a relatively low activation at the softmax layer, as well as some of the TPs. In addition, the FN, i.e., the pixels that should have been S/M 


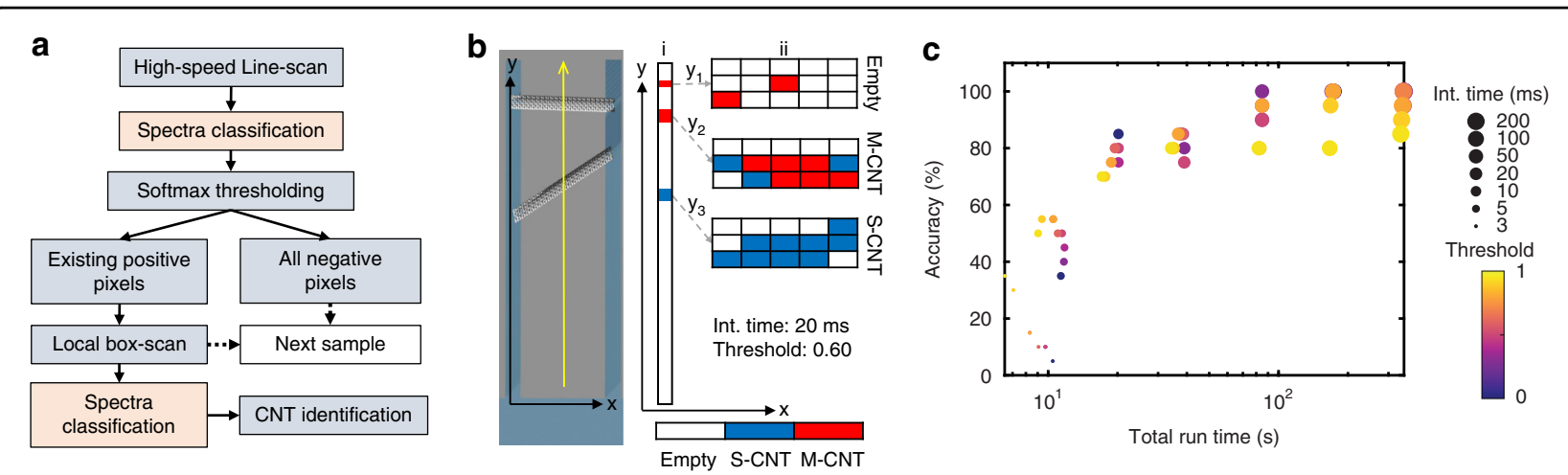

Fig. 5 CNT identification with a 'box-scan' approach. a Workflow of CNT identification. b An example of CNT prediction: (i) high-speed line scan (20 ms/pixel) across a trench and spectral classification: (ii) box-scan ( $20 \mathrm{~ms} /$ pixel) with $3 \times 5$ pixels on the spots of positive pixels in (i) and spectral classification. After analysis, one M-CNT at position $\mathrm{y}_{2}$ and one S-CNT at position $\mathrm{y}_{3}$ are identified. $\mathbf{c}$ Accuracy versus total run time for the scanning of 20 trenches while varying the integration time (marker size) and threshold (color). The same integration time is used for the Line scan and BoxScan.

but are wrongly classified will increase as the criteria to be in the correct class become more stringent with an increasing threshold.

Importantly, the ratio between TP and FP is shown in Fig. 4f for M-CNTs and S-CNTs. The threshold value increases from 0.3 up to 0.99 , and by doing so, the true positive to false-positive ratio improves by a factor of approximately 3 when classifying at an integration time of $5 \mathrm{~ms}$. As a comparison, we also included the same curve for integration times of $3 \mathrm{~ms}$ and $10 \mathrm{~ms}$ in the plot. For higher integration times, the classification accuracy measured by the TP-to-FP ratio increases.

\section{CNT identification}

Although applying a threshold to the softmax output can effectively increase the confidence of the classification, it is still challenging to accurately predict the number and type of CNTs in each trench. Moreover, a map scan with resolution-limited pixel spacing results in prohibitively long measurement times, even for the shortest single-pixel integration times. To address these issues, we propose a proof-of-concept workflow employing an initial line scan to detect CNTs in a trench, followed by an optimized 'box scan' approach that rescans areas of interest for CNT identification (Fig. 5a). From the initial high-speed line scan $(25 \mu \mathrm{m}$ with 75 pixels), the spectra are fed into the network model for classification. After applying a threshold to the output of the softmax layer, each pixel in the line map is classified as either negative (empty) or positive (S-CNT or M-CNT). If all the pixels are classified as negative, a high-speed line scan will take place in the next trench. Otherwise, the laser will move back to the positions of the positive pixels and implement a larger area box-scan $(3 \times 5$ pixels $)$ with the same integration time as in the line scan. Then, these box maps (15 spectra/box) will be input into the CNN for classification. Based on the classification results of the spectra in each box map, the number and type of CNTs in each trench are estimated using two simple criteria: (1) In each box, if the number of positive $\mathrm{N}_{\mathrm{PS}}>2$, one CNT is identified; otherwise, no CNT is assumed to be present in this box. (2) In a CNT box, if the number of M-CNTpredicted pixels $\left(N_{\mathrm{M}}\right)$ is more than those of S-CNTpredicted pixels $\left(N_{S}\right), \mathrm{N}_{\mathrm{M}}>\mathrm{N}_{\mathrm{S}}$, this CNT is assumed to be an M-CNT, or otherwise a S-CNT. These criteria are obtained with the highest prediction accuracies on the samples in Fig. 5c.

Figure 5b shows an example of the CNT prediction. After a high-speed line scan across a trench, spectra are classified using the network model. After classification and softmax thresholding (threshold $=0.6$ ), 5 pixels at 3 spots, $y_{1}, y_{2}$, and $y_{3}$, are classified as positives. Then, larger area box scans with $3 \times 5$ pixels are performed on these three spots in (i), followed by the classification for each box (ii). After analysis, one M-CNT at position $\mathrm{y}_{1}$ and one $\mathrm{S}-\mathrm{CNT}$ at position $\mathrm{y}_{2}$ are identified. To assess the usefulness of this approach, Fig. 5c shows the identification accuracy and total run time (laser exposure time) of the identification process for 20 trenches (containing 15 CNTs) while varying the integration time and threshold. The total run time is the sum of the integration time from the line scan and (conditional) box-scan and therefore depends on both the integration time and threshold of the softmax layer. The overall accuracy increases sharply with integration time from 3 to $10 \mathrm{~ms}$ and then slowly levels off. As a trade-off, the total run time increases with an increase in integration time. For a set integration time, there is an intricate dependence on the threshold value of the softmax layer: for very short integration times, where the ratio of true pos./false pos. classification is low, a higher threshold value will increase both accuracy and reduce measurement time because time-consuming box 
scans returning empty results are avoided. In contrast, for long integration times, a higher threshold will sacrifice the accuracy due to the removal of a fraction of true positives (Fig. 4e) but will still reduce the run time. Consequently, the targeted identification accuracy is a crucial parameter for the choice of an optimal value for both integration time and the threshold value in the softmax layer. It is important to note that from an application point of view, this optimal accuracy target will depend on a potentially complicated cost function taking into account a variety of parameters, such as the dynamics of the mechanical positioning/scanning system, speed of data communication between acquisition software and $\mathrm{CNN}$, resulting hardware-dependent total run time, and the cost function of misidentified CNTs for downstream processes. These parameters can vary widely depending on the application and whether the process is carried out on a research setup (such as the WITec Alpha $300 \mathrm{R}$ employed here) or a dedicated production setup running the $\mathrm{CNN}$ on dedicated hardware in the Raman control loop. Additionally, other techniques, such as compressive Raman spectroscopy $^{33,34}$ and multifocal Raman spectroscopy ${ }^{35,36}$, may give rise to a higher scanning rate and higher SNR improvement.

\section{Conclusion}

In conclusion, we implemented a high-throughput highaccuracy approach to identifying suspended CNTs using a Raman spectroscopy line-scan method and deep learning classification method. With this approach, we significantly increased Raman scanning rates compared to large-scale spectroscopic mapping with human-based spectral postprocessing. Reducing the exposure time to only a few milliseconds minimizes laser-induced sample damage, while an appropriately trained $\mathrm{CNN}$ permits the quantitative identification of CNTs on the growth substrate, providing information about their number, position, and physical properties. We expect our high-throughput approach to be applicable to other emerging nanoscales. It allows the integration of a fast, reliable, quantitative, and spatially resolved nanomaterial identification process within an assembly workflow for the integration of nanoscale materials in technological applications.

\section{Materials and methods}

\section{Sample preparation}

The suspended CNT samples were prepared by the same methods as described in a recent work ${ }^{2,26}$. The forklike substrates were fabricated by using standard photolithography and wet etching on a $\mathrm{SiO}_{2} / \mathrm{Si}$ wafer. After the deposition of iron-loaded ferritin particles (as catalyst precursors) on the fork structures, suspended CNTs were grown by chemical vapor deposition (CVD) in a $\mathrm{CH}_{4} / \mathrm{H}_{2}$ atmosphere at $825^{\circ} \mathrm{C}$ for $30 \mathrm{~min}$. After growth, $\sim 50 \%$ of the forks were successfully bridged with 1-3 CNTs, while the rest of the forks remained empty.

\section{Raman measurements}

We measured Raman spectra across the fork-like substrate using a confocal Raman microscope (WiTec, alpha300 R). A $532 \mathrm{~nm}$ laser was used with a $6001 / \mathrm{mm}$ grating to generate spectra. Wavenumber and pinhole calibration was performed using a standard silicon sample. A long focal length ZEISS $50 \times 0.55$ An NA objective lens was used, yielding a diffraction-limited spot size of $\sim 1.2 \mu \mathrm{m}$ in diameter. To collect a large dataset for network training, we first took a map scan on the silicon finger area with a pixel density of 3 pixels per $\mu \mathrm{m}^{2}$ (in both the $\mathrm{x}$ - and $\mathrm{y}$-directions) using the settings of $1 \mathrm{~mW}$ power and $20 \mathrm{~ms}$ integration time. Then, we set a narrow window region $\left(0.1 \times 5 \mu \mathrm{m}^{2}\right)$ on top of the CNR area and performed map scans with various integration times and powers. The Raman settings for the network training dataset are (power/integration time): $0.1 \mathrm{~mW} / 50 \mathrm{~ms}$, $0.3 \mathrm{~mW} / 50 \mathrm{~ms}, \quad 0.5 \mathrm{~mW} / 50 \mathrm{~ms}, \quad 0.7 \mathrm{~mW} / 50 \mathrm{~ms}, \quad 1 \mathrm{~mW} /$ $50 \mathrm{~ms}, 1.5 \mathrm{~mW} / 50 \mathrm{~ms}, 2 \mathrm{~mW} / 50 \mathrm{~ms}, 3 \mathrm{~mW} / 50 \mathrm{~ms}$, and $1 \mathrm{~mW} / 1 \mathrm{~ms}, \quad 1 \mathrm{~mW} / 3 \mathrm{~ms}, \quad 1 \mathrm{~mW} / 5 \mathrm{~ms}, \quad 1 \mathrm{~mW} / 10 \mathrm{~ms}$, $1 \mathrm{~mW} / 20 \mathrm{~ms}, 1 \mathrm{~mW} / 50 \mathrm{~ms}, 1 \mathrm{~mW} / 70 \mathrm{~ms}, 1 \mathrm{~mW} / 100 \mathrm{~ms}$, $1 \mathrm{~mW} / 150 \mathrm{~ms}, 1 \mathrm{~mW} / 200 \mathrm{~ms}, 1 \mathrm{~mW} / 600 \mathrm{~ms}$. We collected the dataset from an empty sample using the same Raman settings as for the S- and M-CNTs. Finally, we obtained a total of 62,130 labeled training spectra acquired on 20 trenches containing S-CNTs, 21 trenches containing M-CNTs, and 20 trenches where no CNTs were present. In addition, we obtained separately labeled datasets for validation consisting of 48,887 spectra acquired on 10 trenches containing S-CNTs, 11 trenches containing M-CNTs, and 15 trenches where no CNTs were present. In the high-speed line-scan experiment, a Raman map $(0.5 \mu \mathrm{m}$ in the $\mathrm{x}$-direction and $20 \mu \mathrm{m}$ in the $\mathrm{y}$ direction) with 2 lines and 75 pixels per line was scanned in the middle position of each trench. Please note that we needed to set at least 2 lines for each map scan in the WiTec Raman microscope, but only one line was taken for the analysis.

Our Raman system is equipped with three objectives: $10 \times 0.25 \mathrm{NA}, 50 \times 0.55 \mathrm{NA}$, and $100 \times 0.9$. The $10 \times 0.25$ NA objective has a low spatial resolution since its diffraction-limited spot size is as large as $\sim 2.6 \mu \mathrm{m}$ in diameter. Given the length of the finger of the growth substrate of $20 \mu \mathrm{m}$, with this spot size, it would be challenging to distinguish two CNTs that are grown in the same trench within $3 \mu \mathrm{m}$ from each other. On the other hand, the $100 \times 0.9$ NA objective has a much higher spatial resolution of $\sim 0.72 \mu \mathrm{m}$ but a very shallow depth of focus (FWHM $\sim 0.81 \mu \mathrm{m}$ ); it is therefore not suitable for this application as the CNTs are not perfectly straight and may have some bending. We find that the $50 \times 0.55 \mathrm{NA}$ 
objective, with an intermediate diffraction-limited spot size of $\sim 1.2 \mu \mathrm{m}$ and FWHM of the depth of focus being $\sim 3.2 \mu \mathrm{m}$, is a good trade-off.

\section{SNR calculation}

The SNRs of the CNT spectra for each Raman setting (see Supplementary information Fig. S4) is determined from:

$$
\mathrm{SNR}=\frac{\operatorname{Mean}(G)-\text { Mean }(\text { backgroud })}{\Delta(\text { background })},
$$

where $\operatorname{Mean}(G)$ is the average intensity of the $G$ band region (1540-1605 $\mathrm{cm}^{-1}$ ) and Mean(background) is the average intensity at a region $\left(2000-2200 \mathrm{~cm}^{-1}\right)$ where no bands of CNTs occur. $\Delta$ (background) is the standard variation calculated from the same spectrum in the region $2000-2200 \mathrm{~cm}^{-1}$. For our datasets, the SNR variance for each Raman setting is quite high because of the spatial position variance of the CNTs with respect to the focusing position of the laser beam. When intradataset variance is high, a large number of spectra per Raman setting may help to better represent the full data distribution and lead to higher predictive performance.

\section{CNN architecture}

The Raman spectra (dimensions $1600 \times 1$ ) are fed to a neural network in which the layout has been optimized using gridsearch through a various residual neural network (ResNet) and convolutional neural network (CNN) architectures with various hyperparameters (see Supplementary information: Part 2). We found that the optimized network is a regular $\mathrm{CNN}$ consisting of 4 blocks, each consisting of a convolutional layer, a batch normalization layer, and a ReLU activation layer. The convolutional layer contains 192 filters of size $13 \times 1$ that have a stride of 2 and a padding of 6 such that the size of the input is reduced by half $(800,400$, 200 , and 100) after each convolutional layer. The network is completed with a fully connected layer in which the number of neurons matches the number of classes (3), a softmax activation layer and a cross-entropy layer to define the loss function. Finally, we note that for all Raman spectra, cosmic ray removal is performed individually, as well as a standardization step $($ mean $=0$, standard deviation $=1)$.

\section{Acknowledgements}

We acknowledge financial support from Strategic Focus Area (SFA) Advanced Manufacturing (Project NanoAssembly). M.L.P. and J.Z. acknowledge funding by the EMPAPOSTDOCS-II program, which has received funding from the European Union's Horizon 2020 research and innovation program under the Marie Skłodowska-Curie Grant Agreement no. 754364. M.L.P. also acknowledges funding from the Swiss National Science Foundation under Spark grant no. 196795. We thank Binning and Rohrer Nanotechnology Center (IBM ResearchZurich) and ETH FIRST-CLA laboratory teams for technical support.

\section{Author details}

'Laboratory for Transport at Nanoscale Interfaces, Empa, Swiss Federal Laboratories for Materials Science and Technology, CH-8600 Dübendorf,
Switzerland. ${ }^{2}$ Machine Learning and Optimization Laboratory, School of Computer and Communication Sciences, EPFL, CH-1015 Lausanne, Switzerland. ${ }^{3}$ Department of Physics and Swiss Nanoscience Institute, University of Basel, CH-4056 Basel, Switzerland. ${ }^{4}$ Micro- and Nanosystems, Department of Mechanical and Process Engineering, ETH Zurich, CH-8092 Zurich, Switzerland

\section{Author contributions}

M.C., C.H., M.J. and C.R. designed the project. M.L.P., L.B., A.A. and B.G. developed the deep learning algorithms and carried out the classification. J.Z., J.O. and M.H. developed the data collection protocols and collected the datasets. S.H. performed the growth of the materials. R.M. developed the LabView interface. J.Z., R.B. and R.M. developed the scripts for automatic data collection. J.Z., M.L.P. and J.O. wrote the manuscript. All authors discussed the results and implications and commented on the manuscript.

\section{Data availability}

The data are available from the corresponding authors upon reasonable request.

\section{Code availability}

The codes are available from the corresponding authors upon reasonable request.

Conflict of interest

The authors declare no competing interests.

Supplementary information The online version contains supplementary material available at https://doi.org/10.1038/s41378-022-00350-w.

Received: 4 August 2021 Revised: 24 November 2021 Accepted: 5 December 2021

Published online: 10 February 2022

\section{References}

1. Cardenas, J. A., Andrews, J. B., Noyce, S. G. \& Franklin, A. D. Carbon nanotube electronics for loT sensors. Nano Futures 4, 012001 (2020).

2. Jung, S., Hauert, R., Haluska, M., Roman, C. \& Hierold, C. Understanding and improving carbon nanotube-electrode contact in bottom-contacted nanotube gas sensors. Sens. Actuat. B Chem. 331, 129406 (2021).

3. Chikkadi, K, Muoth, M., Maiwald, V., Roman, C. \& Hierold, C. Ultra-low power operation of self-heated, suspended carbon nanotube gas sensors. Appl. Phys. Lett. 103, 223109 (2013).

4. Hills, G. et al. Modern microprocessor built from complementary carbon nanotube transistors. Nature 572, 595-602 (2019).

5. Peng, L. M., Zhang, Z. \& Qiu, C. Carbon nanotube digital electronics. Nat. Electron. 2, 499-505 (2019).

6. Shulaker, M. M. et al. Carbon nanotube computer. Nature 501, 526-530 (2013).

7. Akinwande, D. et al. Graphene and two-dimensional materials for silicon technology. Nature 573, 507-518 (2019).

8. Dresselhaus, M. S., Dresselhaus, G., Saito, R. \& Jorio, A. Raman spectroscopy of carbon nanotubes. Phys. Rep. 409, 47-99 (2005).

9. Farhat, H. et al. Observation of electronic Raman scattering in metallic carbon nanotubes. Phys. Rev. Lett. 107, 157401 (2011).

10. Zhang, D., Yang, J., Li, M. \& Li, Y. ( $(n, m)$ assignments of metallic single-walled carbon nanotubes by Raman spectroscopy: the importance of electronic Raman scattering. ACS Nano 10, 10789-10797 (2016).

11. Ho, C. S. et al. Rapid identification of pathogenic bacteria using Raman spectroscopy and deep learning. Nat. Commun. 10, 4927 (2019).

12. Dieing, T. \& Hollricher, O. High-resolution, high-speed confocal Raman imaging. Vib. Spectrosc. 48, 22-27 (2018).

13. Butler, H. J. et al. Using Raman spectroscopy to characterize biological materials. Nat. protoc. 11, 664-687 (2016).

14. Schmidhuber, J. Deep Learning in neural networks: an overview. Neural Netw. 61, 85-117 (2015)

15. Sun, Y., Wang, X. \& Tang, X. Deep learning face representation from predicting 10,000 classes. In Proc. IEEE Computer Society Conference on Computer Vision and Pattern Recognition, 1891-1898 (IEEE Computer Society, 2014). 
16. Liu, Z., Luo, P., Wang, X. \& Tang, X. Deep learning face attributes in the wild. In IEEE International Conference on Computer Vision (ICCV), 3730-3738 (IEEE Computer Society, 2015).

17. Mikolov, T., Karafiat, M., Burget, L., Cernocky, J. \& Khudanpur, S. Recurrent neural network based language model. In Interspeech, 2, 1045-1048 (International Speech Communication Association, 2010).

18. Hinton, G. et al. Deep neural networks for acoustic modeling in speech recognition: the shared views of four research groups. IEEE Signal Process. Mag. 29, 82-97 (2012).

19. Zhang, X., Zhao, J. \& LeCun, Y. Character-level convolutional networks for text classification. Adv. Neural Inf. Process. Syst. 28, 649-657 (Morgan Kaufmann Publishers Inc., 2015).

20. Tshitoyan, V. et al. Unsupervised word embeddings capture latent knowledge from materials science literature. Nature 571, 95-98 (2019).

21. Cabosart, D. et al. A reference-free clustering method for the analysis of molecular break-junction measurements. Appl. Phys. Lett. 114, 143102 (2019).

22. Abbassi, M. E. et al. Benchmark and application of unsupervised classification approaches for univariate data. Commun. Phys. 4, 50 (2021).

23. Kourou, K., Exarchos, T. P., Exarchos, K. P., Karamouzis, M. V. \& Fotiadis, D. I. Machine learning applications in cancer prognosis and prediction. Comput. Struct. Biotec. 13, 8-17 (2015).

24. Bojarski, M. et al. End to end learning for self-driving cars. arXiv preprint arXiv:1604.07316 (2016).

25. Shin, $H$. et al. Early-stage lung cancer diagnosis by deep learning-based spectroscopic analysis of circulating exosomes. ACS Nano 14, 5435-5444 (2020).

26. Muoth, M. \& Hierold, C. Transfer of carbon nanotubes onto microactuators for hysteresis-free transistors at low thermal budget. In Proceedings of IEEE 25th
International Conference on Micro Electro Mechanical Systems (MEMS), 1352-1355 (IEEE, 2012).

27. Muoth, M. et al. Hysteresis-free operation of suspended carbon nanotube transistors. Nat. Nanotech. 5, 589-592 (2010).

28. Kumar, L., Jenni, L. V., Haluska, M., Roman, C. \& Hierold, C. Mechanical stress relaxation in adhesively clamped carbon nanotube resonators. AIP Adv. 8 , 025118 (2018).

29. Ranjan, V. et al. Clean carbon nanotubes coupled to superconducting impedance-matching circuits. Nat. Commun. 6, 7165 (2015).

30. Waissman, J. et al. Realization of pristine and locally tunable onedimensional electron systems in carbon nanotubes. Nat. Nanotech. 8 , 569-574 (2013).

31. Li, Q., Liu, C., Wang, X. \& Fan, S. Measuring the thermal conductivity of individual carbon nanotubes by the Raman shift method. Nanotechnology 20, 145702 (2009).

32. Zhang, $L$. et al. Effects of intense laser irradiation on Raman intensity features of carbon nanotubes. Phys. Rev. B 65, 073401 (2002)

33. Cebeci, D., Mankani, B. R. \& Ben-Amotz, D. Recent trends in compressive Raman spectroscopy using DMD-based binary detection. J. Imaging 5, 1 (2019).

34. Sturm, B. et al. High-sensitivity high-speed compressive spectrometer for Raman imaging. ACS Photonics 6, 1409-1415 (2019).

35. Okuno, M. \& Hamaguchi, H.-O. Multifocus confocal Raman microspectroscopy for fast multimode vibrational imaging of living cells. Opt. Lett. 35, 4096-4098 (2010).

36. Kong, L. \& Chan, J. A rapidly modulated multifocal detection scheme for parallel acquisition of Raman spectra from a 2-D focal array. Anal. Chem. 86, 6604-6609 (2014) 